\title{
Influence of Oxygen on Zinc Oxide Films Fabricated by Ion-Beam Sputter Deposition
}

\author{
Jin-Cherng Hsu ${ }^{1,2}$ and Yueh-Sheng Chiang ${ }^{1}$ \\ ${ }^{1}$ Department of Physics, Fu Jen Catholic University, Hsinchuang, New Taipei City 24205, Taiwan \\ ${ }^{2}$ Graduate Institute of Applied Science and Engineering, Fu Jen Catholic University, Hsinchuang, \\ New Taipei City 24205, Taiwan
}

Correspondence should be addressed to Jin-Cherng Hsu; 054326@mail.fju.edu.tw

Received 25 June 2013; Accepted 30 July 2013

Academic Editors: Z. Jiang and H. Saxén

Copyright (C) 2013 J.-C. Hsu and Y.-S. Chiang. This is an open access article distributed under the Creative Commons Attribution License, which permits unrestricted use, distribution, and reproduction in any medium, provided the original work is properly cited.

In this study, zinc oxide films were deposited by an ion-beam sputter deposition in various oxygen partial pressures at room temperature. The films changed the structures from amorphous to polycrystalline with increasing the oxygen partial pressure $\left(P_{\mathrm{O}_{2}}\right)$. The optimal $P_{\mathrm{O}_{2}}$ was found at $1.2 \times 10^{-4}$ Torr because the film prepared at the oxygen partial pressure had the lowest resistivity and the highest transparence in the visible light region. The lowest resistivity results from a great number of oxygen vacancy sites formed on the polycrystalline surface as exposed to the atmosphere. Moreover, the film has the highest XRD peak intensity, smallest FWHM diffraction peak, smallest $d$-spacing, and smallest biaxial stress.

\section{Introduction}

$\mathrm{ZnO}$ has a hexagonal wurtzite structure with a wide direct band gap of about $3.4 \mathrm{eV}$. It is naturally an n-type semiconductor due to its deviation from stoichiometry as the presence of oxygen vacancies or zinc interstitials [1]. It is important to fabricate an optimal $\mathrm{ZnO}$ film for development of optoelectronic devices due to its high transparence in the visible light region and its low resistivity [2]. In the previous studies of $\mathrm{Ti}, \mathrm{Zr}, \mathrm{Si}$, and $\mathrm{Al}$ metallic oxide films prepared by ion-beam sputter deposition (IBSD) [3], the oxygen partial pressure $\left(P_{\mathrm{O}_{2}}\right)$ can affect their deposition rates, optical properties, and surface morphologies. Depositing at each optimal $P_{\mathrm{O}_{2}}$, the metallic oxide films have higher transparence, higher refractive indices, lower extinction coefficients, and lower surface roughness. They are all amorphous structures and nonconductive films [4]. In this study, an optimal $P_{\mathrm{O}_{2}}$ for zinc metallic target was also found. However, the as-deposited zinc oxide film is polycrystalline structure and has conductive property due to free electron carrier, which induces the optical band gap shift by the Burstein-Moss (BM) effect [5] and band-gap-narrowing (BGN) effect [6]. The BM effect is the blue-shift phenomenon of the band edge where the Fermi level merges into the conduction band with the increase of the carrier concentration. Yet, the BGN effect is a red-shift phenomenon of the band edge because the electron-electron repulsive interaction and the localization of the electron wave function is weakened by the screening of the potential in the high carrier concentration [7]. Apparently, the band gap shift is affected by the combination of the BM and BGN effects as increasing carrier concentrations. Sakai et al. have also investigated that the carrier concentration strongly affects the optical band gap resulting from the oxygen vacancies controlled by the oxygen flow rate during the growth of the polycrystalline $\mathrm{ZnO}$ film by a reactive plasma deposition [7]. In this study, the optical band gaps and the $\mathrm{ZnO}$ structures were determined by controlling the various oxygen partial pressures during the IBSD at room temperature.

In our previous study of IBSD, the adsorbate, water, and hydroxyl $\left(\mathrm{OH}^{-}\right)$groups are formed on the surface of the as-deposited $\mathrm{ZnO}$ films as exposed to the atmosphere. The influences of the adsorbate on the optical property have been illustrated [8]. Furthermore, the electric properties affected by the adsorbate were also investigated in this study. 


\section{Experiment}

Figure 1 shows schematic drawing of the ion beam sputtering system for this study. The system with a Kaufmann type ion source manufactured by Veeco Inc. was equipped with $3 \mathrm{~cm}$ diameter molybdenum grids. A target mounted on a watercooled copper block was pure zinc metal (99.99\%) slab with a size of $12 \times 10 \times 1 \mathrm{~cm}$. Silicon wafer substrate for XRD measurement and one inch diameter B270 glass for optical measurement were cleaned with ethanol in an ultrasonic bath and blow-dried with dry nitrogen gas and then were mounted on a rotatable holder. The chamber was evacuated to a base pressure of $5 \times 10^{-6}$ Torr by a cryo-pump before deposition. Ar gas was fed into ion source as a working gas at flow rate of $\sim 10 \mathrm{sccm}$. The ion-beam voltage and ion-beam current were kept at $1000 \mathrm{~V}$ and $20 \mathrm{~mA}$, respectively. An incident angle between the ion beam and the surface normal of the zinc target was $45^{\circ}$. The target was presputtered by the Ar ion beam for half an hour to clear the surface in the metallic mode before each deposition. Then, oxygen gas was fed into chamber by mass flow meter during deposition, where $P_{\mathrm{O}_{2}}$ was changed from $2 \times 10^{-5}$ to $2 \times 10^{-4}$ Torr. The as-deposited sample was labeled by $\mathrm{ZnO}-\eta$ as the sample was prepared at $P_{\mathrm{O}_{2}}=$ $\eta \times 10^{-5}$ Torr. The total pressure was regulated at about $3 \times$ $10^{-4}$ Torr by feeding Ar gas into ion source during deposition.

The total transmittance spectrum $\left(T_{\text {sub }}\right)$ of the substrate was measured by Varian Cary-5E spectrometer before mounting. The B270 glass substrate has two surfaces. Each surface has the same transmittance $\left(T_{s}\right)$. An incident light from the left side of the substrate is repeatedly reflected between the two surfaces schematically shown in Figure 2. Then, the transmittance $T_{\text {sub }}$, the sum of the incoherent irradiances at the right side of substrate, can be deduced below [9]:

$$
\begin{aligned}
T_{\text {sub }} & =T_{s} T_{s}+T_{s} R_{s} R_{s} T_{s}+T_{s} R_{s} R_{s} T_{s} R_{s} R_{s}+\cdots \\
& =T_{s}^{2}\left(1+R_{s}^{2}+R_{s}^{4}+\cdots\right) \\
& =\frac{T_{s}^{2}}{1-R_{s}^{2}} \\
& =\frac{T_{s}^{2}}{1-\left(1-T_{s}\right)^{2}} \\
& =\frac{1}{2 / T_{s}-1},
\end{aligned}
$$

where $R_{s}$ is the reflection of the single surface and equals ( $1-$ $T_{s}$ ) due to its free absorption. Then, $T_{s}$ can be represented by

$$
T_{s}=\frac{2}{1 / T_{\text {sub }}+1} .
$$

If the as-deposited $\mathrm{ZnO}$ is a low optical absorption material in the visible region, the transmittance of the film deposited on the one of substrate's surface is $T_{f}$; then

$$
T_{f} \fallingdotseq \frac{1}{\left(1 / T_{d}-1 / T_{s}+1\right)} .
$$

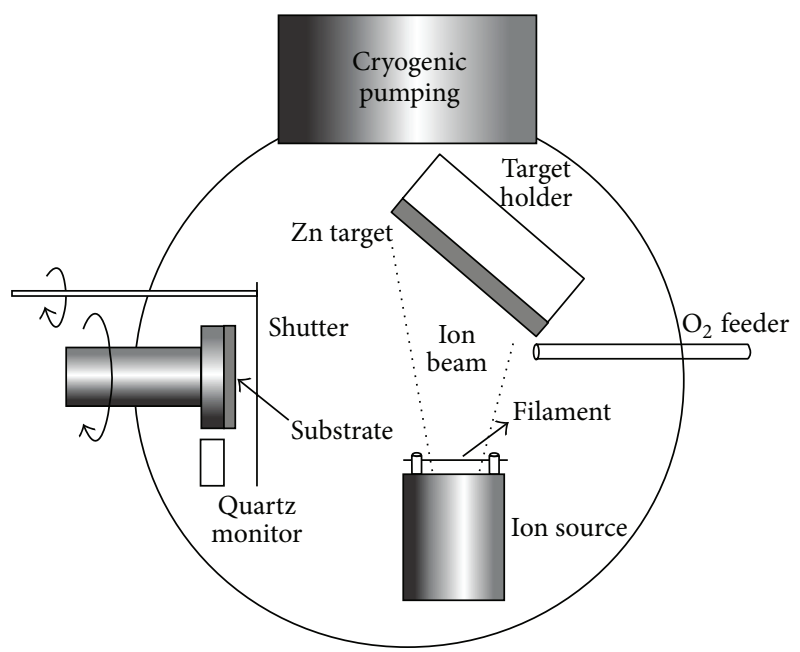

FIGURE 1: Schematic drawing of the ion-beam sputtering system.

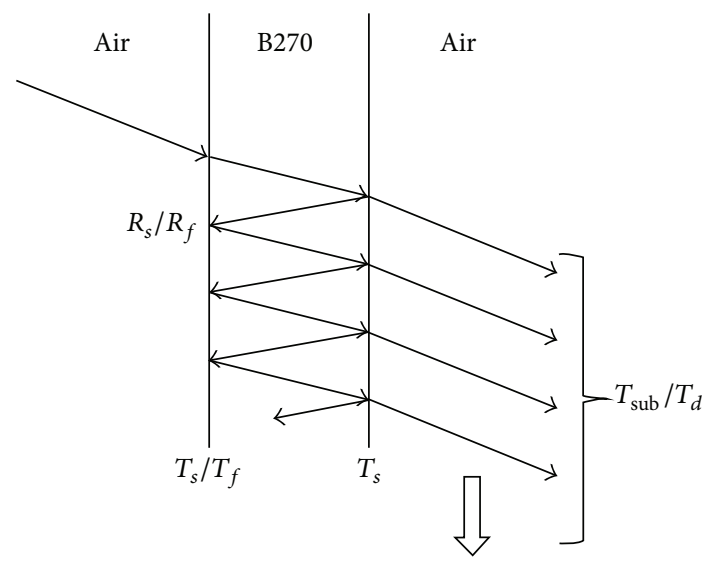

FIgURE 2: The two relative transmittances, $T_{\text {sub }}$ and $T_{d}$, are schematically shown by the sums of the incoherent irradiances in the right side of the B270 substrate, where $T_{s}$ and $T_{f}$ are the transmissions of the uncoated and coated surfaces of the substrate and $R_{s}$ and $R_{f}$ are the reflectance of the two surfaces, respectively.

In (3), $T_{d}$ is the transmittance measured by Cary $5 \mathrm{E}$ spectrometer after deposition and is also the sum of the incoherent irradiances in the right side of the substrate including the repeatedly reflected light schematically shown in Figure 2. It can be similarly deducted from (1). Minus values of $1 / T_{s}$ in (3) reduce the irradiances interference from the other uncoated substrate's surface. Measuring the transmittances of $T_{\text {sub }}$ and the $T_{d}$ and calculating the transmittances by the above three formulas, the optical absorption $(\alpha)$ of the $\mathrm{ZnO}$ film is more accurately evaluated by the equation of BeerLambert's law [10], $T_{f}=\exp (-\alpha t)$, where small capital $t$ is the film's thickness.

The surface morphologies of the films were investigated by an atomic force microscope, Dimension 3100 made by Digital Instruments. The charge carrier concentrations, sheet resistivities, and the mobilities of the $\mathrm{ZnO}$ films were measured by the van der Pauw method with an HL55WIN Hall System. The crystalline structures were characterized by 
TABLE 1: The resistivities and thickness of the films deposited at various oxygen partial pressures.

\begin{tabular}{lccc}
\hline Sample & $\begin{array}{c}\text { Sheet resistivity } \\
(\Omega / \text { square })\end{array}$ & $\begin{array}{c}\text { Thickness } \\
(\mathrm{nm})\end{array}$ & $\begin{array}{c}\text { Resistivity } \\
(\mathrm{m} \Omega \cdot \mathrm{cm})\end{array}$ \\
\hline $\mathrm{ZnO}-2$ & $1860 \pm 50$ & 96.1 & $13.7 \pm 0.4$ \\
$\mathrm{ZnO}-4$ & $1500 \pm 400$ & 94.2 & $10.6 \pm 2.8$ \\
$\mathrm{ZnO}-8$ & $940 \pm 20$ & 102.5 & $7.40 \pm 0.37$ \\
$\mathrm{ZnO}-10$ & $400 \pm 20$ & 86.9 & $2.64 \pm 0.16$ \\
$\mathrm{ZnO}-12$ & $256 \pm 3$ & 122.0 & $2.40 \pm 0.02$ \\
$\mathrm{ZnO}-15$ & $368 \pm 7$ & 117.0 & $3.31 \pm 0.06$ \\
$\mathrm{ZnO}-20$ & $480 \pm 10$ & 114.9 & $4.22 \pm 0.13$ \\
\hline
\end{tabular}

using a $\mathrm{Cu} \mathrm{K}_{\alpha}$ line $(\lambda=1.54055 \AA)$ in X-ray diffractometer made by Rigaku Multiflex. An M-2000 spectroscopic ellipsometer made by J. A. Woollam Co. was used to measure the thicknesses of the thin $\mathrm{ZnO}$ films listed in Table 1.

\section{Results and Discussion}

Figure 3 shows that oxygen partial pressures affect the deposition rates of $\mathrm{ZnO}$ films. The rate begins to decrease at $P_{\mathrm{O}_{2}}>$ $2 \times 10^{-5}$ Torr as zinc suboxide forming on the $\mathrm{Zn}$ metallic target surface [4]. As soon as $P_{\mathrm{O}_{2}} \geq 1.0 \times 10^{-4}$ Torr, oxidization layer of zinc metal formed on the target surface decreases the sputtering yield. A sharp drop of deposition rate is about three times less than the rare at the low $P_{\mathrm{O}_{2}}$. Castellano [11] also has explained this phenomenon by ideal gas theory to predict the sharp drop of deposition rate in narrow range of $P_{\mathrm{O}_{2}}$ at which a film forms from metal to oxide. The basic mechanism of this transition is the trapping of oxygen molecules on the target surface [12]. Figure 3 shows that the deposition rate similarly decreases and the surface roughness of $\mathrm{ZnO}$ film decreases with increasing $P_{\mathrm{O}_{2}}$ because each deposited particle averages higher momentum transport rate from the energetic argon ion beam at the lower deposition rate. Then, the deposited particles with the higher surface mobility aggregate smoothly on the substrate.

3.1. Optical Properties. Figure 4 shows the optical transmittance spectra of the $\mathrm{ZnO}$ films between 250 and $750 \mathrm{~nm}$. The transmittances of $\mathrm{ZnO}-2$ to -8 films deposited at $P_{\mathrm{O}_{2}}<$ $1.0 \times 10^{-4}$ Torr are lower than $80 \%$ in visible region due to the heavier optical absorption of the suboxide film. The transmittances of $\mathrm{ZnO}-10$ to -20 deposited at lower deposition rate at $P_{\mathrm{O}_{2}} \geq 1.0 \times 10^{-4}$ Torr are larger than $80 \%$ as the transparent films approach to stoichiometric $[13,14]$. However, the transmittances decrease sharply at absorption edges of about $350 \mathrm{~nm}$ wavelength. When increasing $P_{\mathrm{O}_{2}}$, the red-shift phenomena of the absorption edges obviously happen at the $\mathrm{ZnO}-10$ to -20 samples. For describing precisely, the optical band gap $\left(E_{G}\right)$ of $\mathrm{ZnO}$ film is determined by the equation of $\alpha h v=A\left(h v-E_{G}\right)^{1 / 2}$ for the direct transition, where $A, h \nu$, and $\alpha$ are a constant, the incident photon energy, and the absorption coefficient, respectively. The absorption coefficient is calculated by Beer-Lambert's law with the equation of $\alpha=-\ln \left(T_{f}\right) / t$ [14]. Then, $E_{g} s$

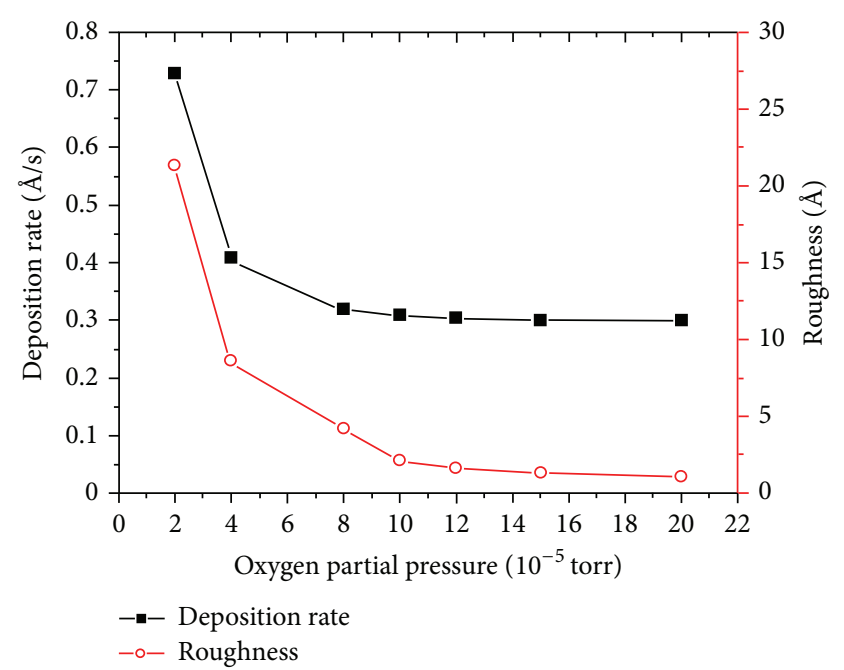

Figure 3: Deposition rates and surface roughness of the $\mathrm{ZnO}$ films versus oxygen partial pressure.

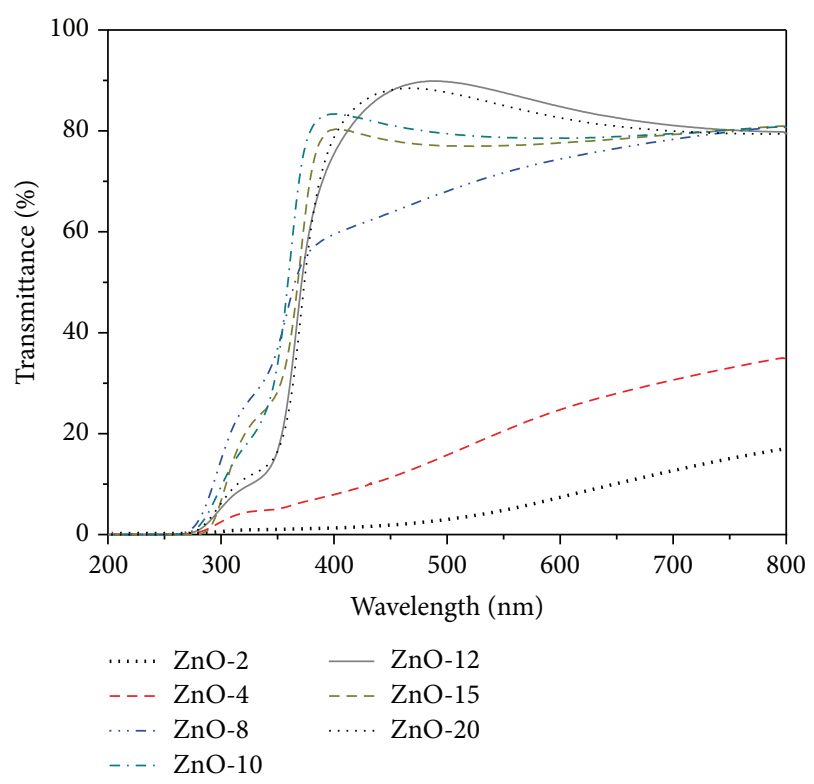

Figure 4: Transmittance of the $\mathrm{ZnO}$ films deposited at different oxygen partial pressures.

are obtained by Tauc's plot [15] with extrapolating the linear portion to the photon energy axis as plotted the $(\alpha h \nu)^{2}$ versus $h \nu$ in Figure 5. The absorption edges red-shift from 3.41 to $3.31 \mathrm{eV}$ with increasing $P_{\mathrm{O}_{2}}$.

The red-shift phenomenon is also proved by Tan et al. They have fabricated the $\mathrm{ZnO}$ films by metal organic chemical vapor deposition at the growth temperatures from 200 to $500^{\circ} \mathrm{C}$ and found their films transfer from amorphous to polycrystalline structure at the treatment processes [16]. Sun et al. have also studied that the $E_{G}$ has a close relationship with the amount of oxygen vacancy sites that induce the electron carriers in the polycrystalline $\mathrm{ZnO}$ film deposited by laser ablating $\mathrm{Zn}$ target in various $P_{\mathrm{O}_{2}}$ [17]. In our study, the $\mathrm{ZnO}-2$ to -8 films belong to amorphous structures, and 


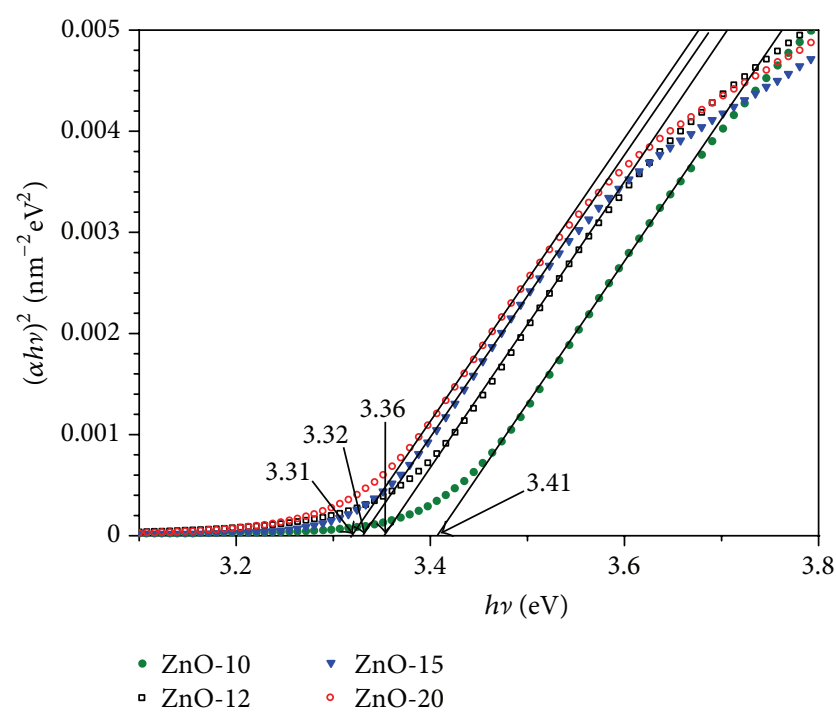

Figure 5: The optical band gaps of the $\mathrm{ZnO}-10$ to -20 samples determined by extrapolating the linear part of the curve to the photon energy axis.

the polycrystalline structures form in the $\mathrm{ZnO}-10$ to -20 films illustrated by XRD as shown in Figure 6. The structures gradually transfer from amorphous to polycrystalline structure with increasing $P_{\mathrm{O}_{2}}$ during deposition. The $\mathrm{ZnO}-10$ structure belongs to the mixture of the amorphous and polycrystalline structures because a little XRD peak begins to grow at $P_{\mathrm{O}_{2}}$ of $1 \times 10^{-4}$ Torr. That is indirectly proved by the fact that its $E_{G}$ of $3.41 \mathrm{eV}$ is larger than that of zinc oxide material having a polycrystalline structure with a direct band gap of about $3.37 \mathrm{eV}$ [18]. Because the polycrystalline structure has oxygen vacancy sites to induce the electron carriers, the amount of the carrier also increases with increasing $P_{\mathrm{O}_{2}}$. Until $P_{\mathrm{O}_{2}}>1.5 \times 10^{-4}$ Torr, the amount decreases again due to the fact that oxygen atoms occupy their oxygen vacancies during deposition. Illustrating ZnO-10 to -15 samples from Figures 5 and 7, the absorption edges red-shift from 3.41 to $3.32 \mathrm{eV}$ with respect to the increase sheet concentrations from $3.01 \times 10^{15}$ to $1.82 \times 10^{16} \mathrm{~cm}^{-2}$ with increasing $P_{\mathrm{O}_{2}}$. The red-shift phenomenon is the electron-electron repulsive interaction from the many electron carriers due to the bandgap-narrowing (BGN) effect [7], that is, the optical band gaps red-shift resulting from the increases of electron carrier concentrations in the films. However, the sheet concentration reduces to $6.15 \times 10^{15} \mathrm{~cm}^{-2}$; the optical band gap still slightly decreases to $3.31 \mathrm{eV}$ due to $\mathrm{BM}$ effect at $P_{\mathrm{O}_{2}}=2.0 \times 10^{-4}$ Torr. Lu et al. [19] and Sakai et al. [7] have mentioned that the carrier concentration in the film affects the shift of the optical absorption edge for $\mathrm{ZnO}$ films in both the $\mathrm{BM}$ effect and the BGN effect as a function of $E_{G}=E_{0}+\Delta E_{\mathrm{BM}}-\Delta E_{\mathrm{BGN}}$, where $E_{G}$ described the optical band gap as the minimum energy needed to excite an electron from the valence band to the conduction band. $E_{0}$ is the energy gap between the conduction and valence band edges in the pure undoped crystal. $\Delta E_{\mathrm{BM}}$ is the energy blue-shift due to BM effect. $\Delta E_{\mathrm{BGN}}$ is the energy red-shift due to BGN effect. The band gap shift

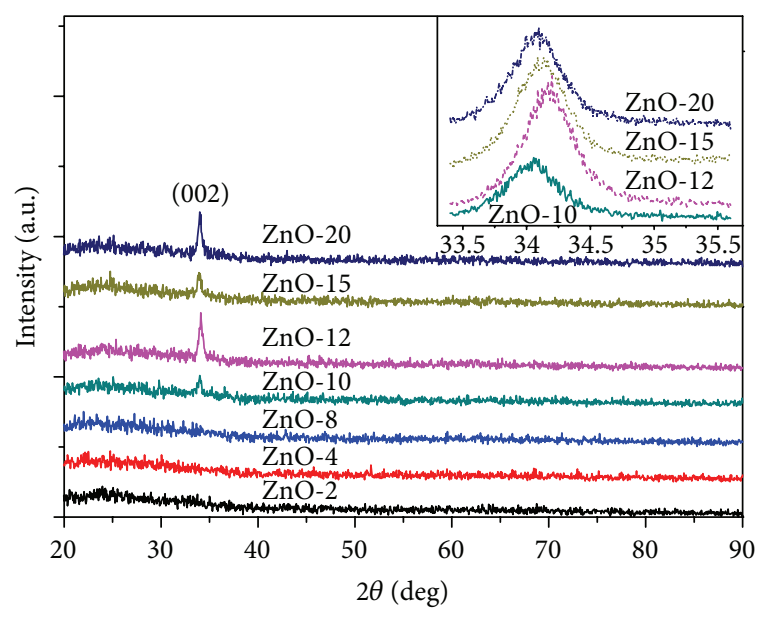

FIGURE 6: The (002) peaks of XRD clearly measured from $\mathrm{ZnO}-10$ to -20 films.

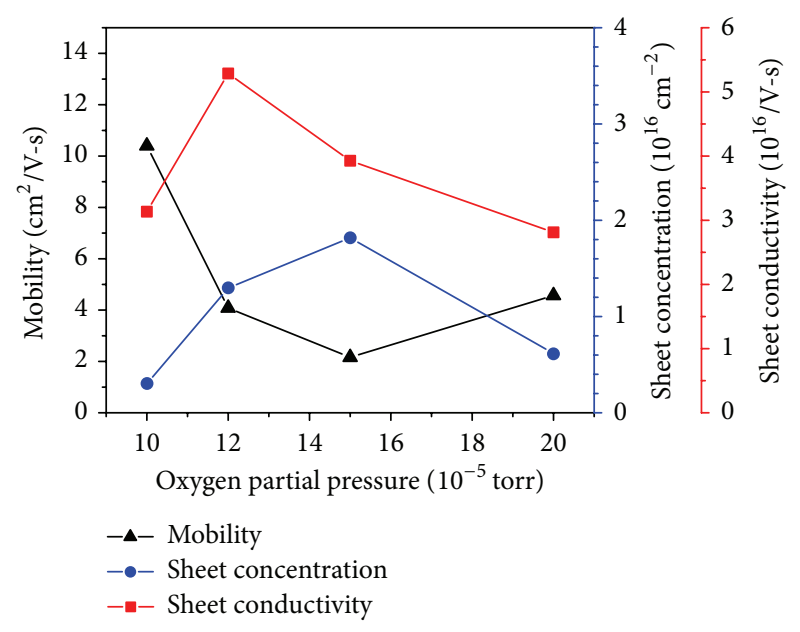

FIGURE 7: Mobility, sheet concentration, and sheet resistivities of the $\mathrm{ZnO}$ films plotted as a function of oxygen partial pressure.

determined by a combination of the two effects is a function of $P_{\mathrm{O}_{2}}$. Thus, there is a critical $P_{\mathrm{O}_{2}}, 1.5 \times 10^{-4}$ Torr, to determine the band gap $\left(E_{G}\right)$ blue- or red-shift.

3.2. Electrical Properties. Figure 7 shows the measurements of Hall effect for the mobility and sheet concentration of the $\mathrm{ZnO}$ films. The sheet concentrations increase with increasing $P_{\mathrm{O}_{2}}$ until $1.2 \times 10^{-4}$ Torr, because the electron carriers are induced by the increasing amount of oxygen vacancies and intrinsic defects of $\mathrm{ZnO}$ films. The mobilities decrease from $10.4,4.08$ to $2.16 \mathrm{~cm}^{2} / \mathrm{V}$.s because of the electron scattering and grain-boundary scattering in polycrystalline film [20]. Yet, the sheet conductivity is proportional to the product of the mobility and carrier concentration of electrons if the conductivity is primarily due to electron. In the absence of the largest amount of sheet concentration and mobility, $\mathrm{ZnO}-12$ film still has the highest sheet conductivity. Because the sheet resistivity is defined as the inverse of the sheet conductivity, $\mathrm{ZnO}-12$ has the least sheet resistivity of $256 \Omega$ /square as 


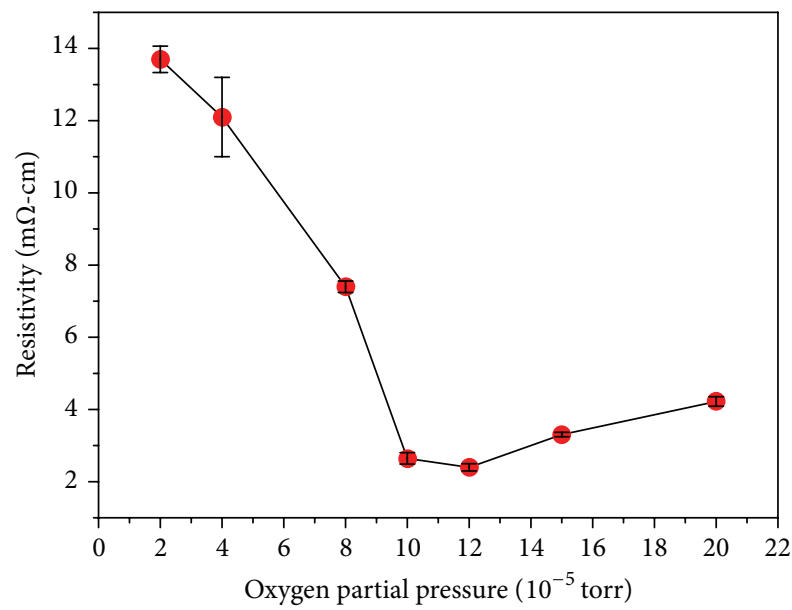

Figure 8: Resistivities of the $\mathrm{ZnO}$ films plotted as a function of oxygen partial pressure.

shown in Table 1. Figure 8 also shows that the resistivities decrease as $P_{\mathrm{O}_{2}}$ increasing until $1.2 \times 10^{-4}$ Torr and increase again as $P_{\mathrm{O}_{2}}>1.2 \times 10^{-4}$ Torr, and $\mathrm{ZnO}-12$ has the least resistivity of $2.4 \mathrm{~m} \Omega \cdot \mathrm{cm}$.

Nevertheless, the decrease of the sheet concentration of $\mathrm{ZnO}-20$ deposited at higher $P_{\mathrm{O}_{2}}=2.0 \times 10^{-4}$ Torr is attributed to improvement of the stoichiometry that may decrease the concentration of the oxygen vacancies and intrinsic defects. Moreover, the mobility increases a little again at the $P_{\mathrm{O}_{2}}$ as shown in Figure 7. It is for that the electron scattering dominates the mobilities of films resulting from the nearly invariable gain-boundary scattering on the almost same grain sizes of the films described by XRD in the following section.

3.3. XRD Measurement of the $\mathrm{ZnO}$ Films. It seems that the electrical property is not only attributed to the film's oxidation. $\mathrm{ZnO}$ film has a strong tendency for self-organized growth as the film has a strong preferred $c$-axis growth orientation [15]. When the films are about $100 \mathrm{~nm}$ thick prepared at $P_{\mathrm{O}_{2}}>1.0 \times 10^{-4}$ Torr, the films obviously have (002) XRD peaks locating at double diffraction angle $(2 \theta)$ around $34.17^{\circ}$ as shown in Figure 6. Table 2 shows the fitted results of the XRD peaks with the intensity, double diffraction angle $(2 \theta)$, and FWHM $(\beta)$. The diffraction angle $(\theta)$ can be represented by $d$-spacing $(d)$, between the atomic (002) planes, calculated by Bragg's diffraction equation, $2 d \sin \theta=$ $\lambda$, and the FWHM can be represented by grain size $(s)$ of the $\mathrm{ZnO}$ crystal, by Scherrer's formula [21], $s=0.9 \lambda / \beta \cos \theta$ as a function of $P_{\mathrm{O}_{2}}$ shown in Figure 9. According to the results of Figures 8 and 9 , the higher the crystallinity is the lower the resistivity at $P_{\mathrm{O}_{2}} \geq 1.2 \times 10^{-4}$ Torr is. The correlation between the most intensity of XRD and the lowest resistivity of $2.4 \mathrm{~m} \Omega \cdot \mathrm{cm}$ for $\mathrm{ZnO}-12$ sample definitely indicates that the crystalline structure plays an important role in the electrical behavior of the $\mathrm{ZnO}$ film. Besides, the crystalline $\mathrm{ZnO}-12$ film has the least $d$ of $2.623 \AA$ and the largest $s$ of $17.5 \mathrm{~nm}$ shown in Figure 9 due to the best crystallization at the highest diffraction angle, $2 \theta=34.17^{\circ}$, shown in Table 2 .

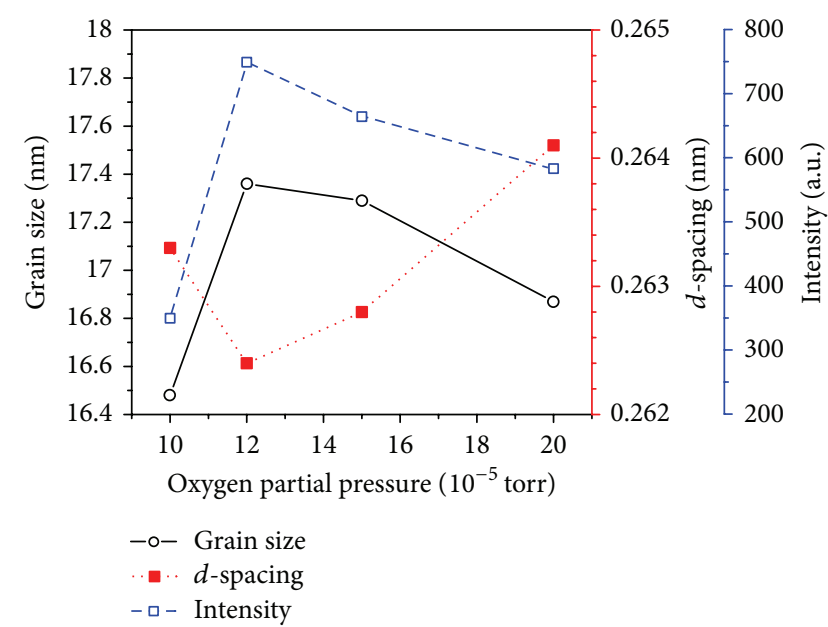

FIgURE 9: The XRD intensities, $d$-spacings, and grain sizes of the $\mathrm{ZnO}-10$ to -20 samples versus oxygen partial pressure.

TABLE 2: The XRD spectra of the $\mathrm{ZnO}$ samples, from $\mathrm{ZnO}-10$ to $\mathrm{ZnO}-20$, fitted by Gauss functions and represented by intensities, double diffraction angles, and FHWMs.

\begin{tabular}{lccc}
\hline Sample & Intensity (a. u.) & $2 \theta(\mathrm{deg})$ & FWHM (deg) \\
\hline $\mathrm{ZnO}-10$ & 349.5 & 34.04 & 0.505 \\
$\mathrm{ZnO}-12$ & 749.1 & 34.17 & 0.479 \\
$\mathrm{ZnO}-15$ & 664.2 & 34.12 & 0.481 \\
$\mathrm{ZnO}-20$ & 583.0 & 34.08 & 0.493 \\
\hline
\end{tabular}

Besides, the biaxial strains of the $\mathrm{ZnO}$ films in $c$-axis direction are determined by the previous XRD as shown in Figure 10. The residual stress $(\sigma)$ based on the biaxial strain model was calculated by the formula, $\sigma=-233\left(c-c_{0}\right) / c_{0} \mathrm{GPa}$, where $c$, the lattice constant of $\mathrm{ZnO}$ film, was calculated by the following equation [22]:

$$
\frac{1}{d^{2}}=\frac{4}{3}\left(\frac{h^{2}+h k+k^{2}}{a^{2}}\right)+\frac{l^{2}}{c^{2}},
$$

and $c_{0}$ is the unstrained lattice parameter of $5.205 \AA$ from a $\mathrm{ZnO}$ powder sample [23], where $h, k$, and $l$ are Miller indices of the $\mathrm{ZnO}$ (002) crystallinity. $\mathrm{ZnO}-12$ film also has the least biaxial stress of $-1.848 \mathrm{GPa}$, which indicates that the film has a much denser crystalline structure than the others [24]. The narrowest $d$-spacing, largest grain, size and denser structure of $\mathrm{ZnO}-12$ film may have the advantage of reducing the electron conduction path in the polycrystalline structure and decreasing the resistivity.

Furthermore, water molecular is easily absorbed by oxygen vacancy sites on the $\mathrm{ZnO}$ film $[5,25]$. The electric property may relate to not only the polycrystalline structure but also to the adsorbate on the surface. Schoenes et al. have reported that $\mathrm{ZnO}$ film had a larger conductivity resulting from oxygen vacancy sites or $\mathrm{Zn}$ interstitial atoms [24, 26]. $\mathrm{ZnO}-12$ has the highest XRD peak, largest grains, and denser structure. Exposing the $\mathrm{ZnO}-12$ film to the atmosphere, the oxygen vacancy and interstitial sites on the crystalline 


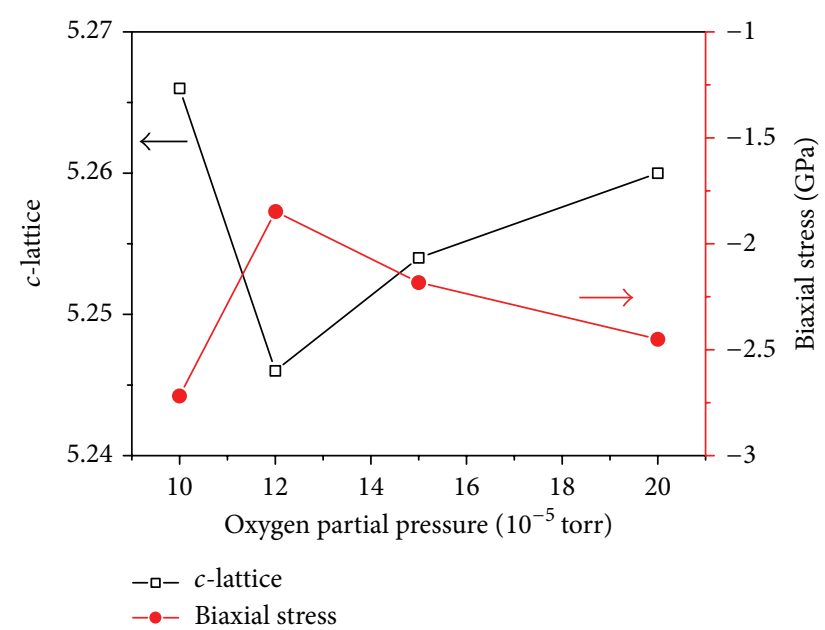

Figure 10: The $c$-lattice constants and biaxial stresses of the $\mathrm{ZnO}-10$ to -20 samples ploted as a function of oxygen partial pressure.

surface absorbed the water and hydroxyl groups. The greater amounts of the absorbents cover the surface of $\mathrm{ZnO}-12$ film as the two subpeaks locating at the binding energies of 532.7 and $531.7 \mathrm{eV}$ have the largest areas in $\mathrm{O}_{1 \mathrm{~s}}$ XPS spectra. That has been proved by our previous study [8]. It is deduced that the $\mathrm{ZnO}$ crystalline formation and oxygen vacancy sites on the $\mathrm{ZnO}$ surface is associated with oxygen partial pressure during deposition, and the electron carriers produced by this crystalline structure benefit lower resistance of $\mathrm{ZnO}$ film as exposed to the atmosphere.

\section{Conclusion}

In this study, optical and electronic properties of $\mathrm{ZnO}$ films were controlled by changing oxygen partial pressure in IBSD of zinc metallic target with ion-beam voltage of $1000 \mathrm{~V}$ and ion-beam current of $20 \mathrm{~mA}$ at room temperature. The optical band gaps of the as-deposited $\mathrm{ZnO}$ films are influenced by the two BM and BGN effects. Moreover, comparing the optical and electronic properties, an optimal $P_{\mathrm{O}_{2}}$ was found at $1.2 \times$ $10^{-4}$ Torr. At the optimal $P_{\mathrm{O}_{2}}$, the $\mathrm{ZnO}$ film has the highest optical transmittance and the lowest resistivity of $2.4 \mathrm{~m} \Omega \cdot \mathrm{cm}$. The low resistivity attributes to water and hydroxyl groups absorbed by a great number of oxygen vacancy sites on the surface of the polycrystalline structure which has the highest XRD peak intensity, the smallest FWHM of diffraction peak, the least $d$-spacing, and the least biaxial stress. This study for finding the optimal oxygen partial pressure of IBSD also benefits the fabrication of aluminum-doped $\mathrm{ZnO}$ film [27].

\section{Acknowledgment}

The authors acknowledge the sponsorship of the National Science Council of Taiwan under Grant no. NSC102-2221-E030-011.

\section{References}

[1] T. Yamamoto and H. Katayama-Yoshida, "Solution using a codoping method to unipolarity for the fabrication of $p$-type
ZnO, Japanese Journal of Applied Physics, vol. 38, no. 2, pp. L166-L169, 1999.

[2] E. Budianu, M. Purica, F. Iacomi, C. Baban, P. Prepelita, and E. Manea, "Silicon metal-semiconductor-metal photodetector with zinc oxide transparent conducting electrodes," Thin Solid Films, vol. 516, no. 7, pp. 1629-1633, 2008.

[3] C.-C. Lee, D. T. Wei, J.-C. Hsu, and C.-H. Shen, "Influence of oxygen on some oxide films prepared by ion beam sputter deposition," Thin Solid Films, vol. 290-291, pp. 88-93, 1996.

[4] C.-C. Lee, J.-C. Hsu, and D.-H. Wong, "Characteristics of some metallic oxides prepared in high vacuum by ion beam sputtering," Applied Surface Science, vol. 171, no. 1-2, pp. 151-156, 2001.

[5] E. Burstein, "Anomalous optical absorption limit in InSb," Physical Review, vol. 93, no. 3, pp. 632-633, 1954.

[6] A. P. Roth, J. B. Webb, and D. F. Williams, "Band-gap narrowing in heavily defect-doped ZnO," Physical Review B, vol. 25, no. 12, pp. 7836-7839, 1982.

[7] K. Sakai, T. Kakeno, T. Ikari et al., "Defect centers and optical absorption edge of degenerated semiconductor $\mathrm{ZnO}$ thin films grown by a reactive plasma deposition by means of piezoelectric photothermal spectroscopy," Journal of Applied Physics, vol. 99, no. 4, Article ID 043508, 7 pages, 2006.

[8] J.-C. Hsu, Y.-H. Lin, P. W. Wang, and Y.-Y. Chen, "Spectroscopic ellipsometry studies on various zinc oxide films deposited by ion beam sputtering at room temperature," Applied Optics, vol. 51, no. 9, pp. 1209-1215, 2012.

[9] H. A. Macleod, Thin-Film Optical Filters, CRC Press, Taylor \& Francis, 4th edition, 2010.

[10] W. T. Lim and C. H. Lee, "Highly oriented $\mathrm{ZnO}$ thin films deposited on Ru/Si substrates," Thin Solid Films, vol. 353, no. 1, pp. 12-15, 1999.

[11] R. N. Castellano, "Reactive ion beam sputtering of thin films of lead, zirconium and titanium," Thin Solid Films, vol. 46, no. 2, pp. 213-221, 1977.

[12] C.-C. Lee, J.-C. Hsu, D. T. Wei, and J.-H. Lin, "Morphology of dual beam ion sputtered films investigated by atomic force microscopy," Thin Solid Films, vol. 308-309, no. 1-4, pp. 74-78, 1997.

[13] N. A. Suvorova, I. O. Usov, L. Stan et al., "Structural and optical properties of $\mathrm{ZnO}$ thin films by rf magnetron sputtering with rapid thermal annealing," Applied Physics Letters, vol. 92, no. 14, Article ID 141911, 2008.

[14] N. Mehan, V. Gupta, K. Sreenivas, and A. Mansingh, "Effect of annealing on refractive indices of radio-frequency magnetron sputtered waveguiding zinc oxide films on glass," Journal of Applied Physics, vol. 96, no. 6, pp. 3134-3139, 2004.

[15] J. Tauc, R. Grigorovici, and A. Vancu, "Optical properties and electronic structure of amorphous germanium," Physica Status Solidi B, vol. 15, no. 2, pp. 627-637, 1966.

[16] S. T. Tan, B. J. Chen, X. W. Sun et al., "Blueshift of optical band gap in $\mathrm{ZnO}$ thin films grown by metal-organic chemical-vapor deposition," Journal of Applied Physics, vol. 98, no. 1, Article ID 013505, 2005.

[17] L. Sun, W. Cheng, F. Lin, X. Ma, and W. Shi, "Changes of structure and optical energy gap induced by oxygen pressure during the deposition of ZnO films," Physica B, vol. 381, no. 1-2, pp. 109112, 2006.

[18] M.-H. Zhao, Z.-L. Wang, and S. X. Mao, "Piezoelectric characterization individual zinc oxide nanobelt probed by piezoresponse force microscope," Nano Letters, vol. 4, no. 4, pp. 587590, 2004. 
[19] J. G. Lu, S. Fujita, T. Kawaharamura et al., "Carrier concentration dependence of band gap shift in n-type $\mathrm{ZnO}: \mathrm{Al}$ films," Journal of Applied Physics, vol. 101, no. 8, Article ID 083705, 2007.

[20] A. F. Mayadas and M. Shatzkes, "Electrical-resistivity model for polycrystalline films: the case of arbitrary reflection at external surfaces," Physical Review B, vol. 1, no. 4, pp. 1382-1389, 1970.

[21] B. D. Cullity, Elements of X-Ray Diffractions, Addison-Wesley, Reading, Mass, USA, 1978.

[22] C. S. Barret and T. B. Massalski, Structure of Metals, Pergamon Press, Oxford, UK, 1980.

[23] R. Cebulla, R. Wendt, and K. Ellmer, "Al-doped zinc oxide films deposited by simultaneous $\mathrm{rf}$ and dc excitation of a magnetron plasma: relationships between plasma parameters and structural and electrical film properties," Journal of Applied Physics, vol. 83, no. 2, pp. 1087-1095, 1998.

[24] J. Schoenes, K. Kanazawa, and E. Kay, "Band and hopping conduction in high-resistivity ZnO," Journal of Applied Physics, vol. 48, no. 6, pp. 2537-2542, 1977.

[25] A. Önsten, D. Stoltz, P. Palmgren, S. Yu, M. Göthelid, and U. O. Karlsson, "Water adsorption on $\mathrm{znO}(0001)$ : transition from triangular surface structures to a disordered hydroxyl terminated phase," Journal of Physical Chemistry C, vol. 114, no. 25, pp. 11157-11161, 2010.

[26] M. Suchea, S. Christoulakis, N. Katsarakis, T. Kitsopoulos, and G. Kiriakidis, "Comparative study of zinc oxide and aluminum doped zinc oxide transparent thin films grown by direct current magnetron sputtering," Thin Solid Films, vol. 515, no. 16, pp. 6562-6566, 2007.

[27] Y.-Y. Chen, J.-C. Hsu, P. W. Wang, Y.-W. Pai, C.-Y. Wu, and Y.H. Lin, "Dependence of resistivity on structure and composition of AZO films fabricated by ion beam co-sputtering deposition," Applied Surface Science, vol. 257, no. 8, pp. 3446-3450, 2011. 

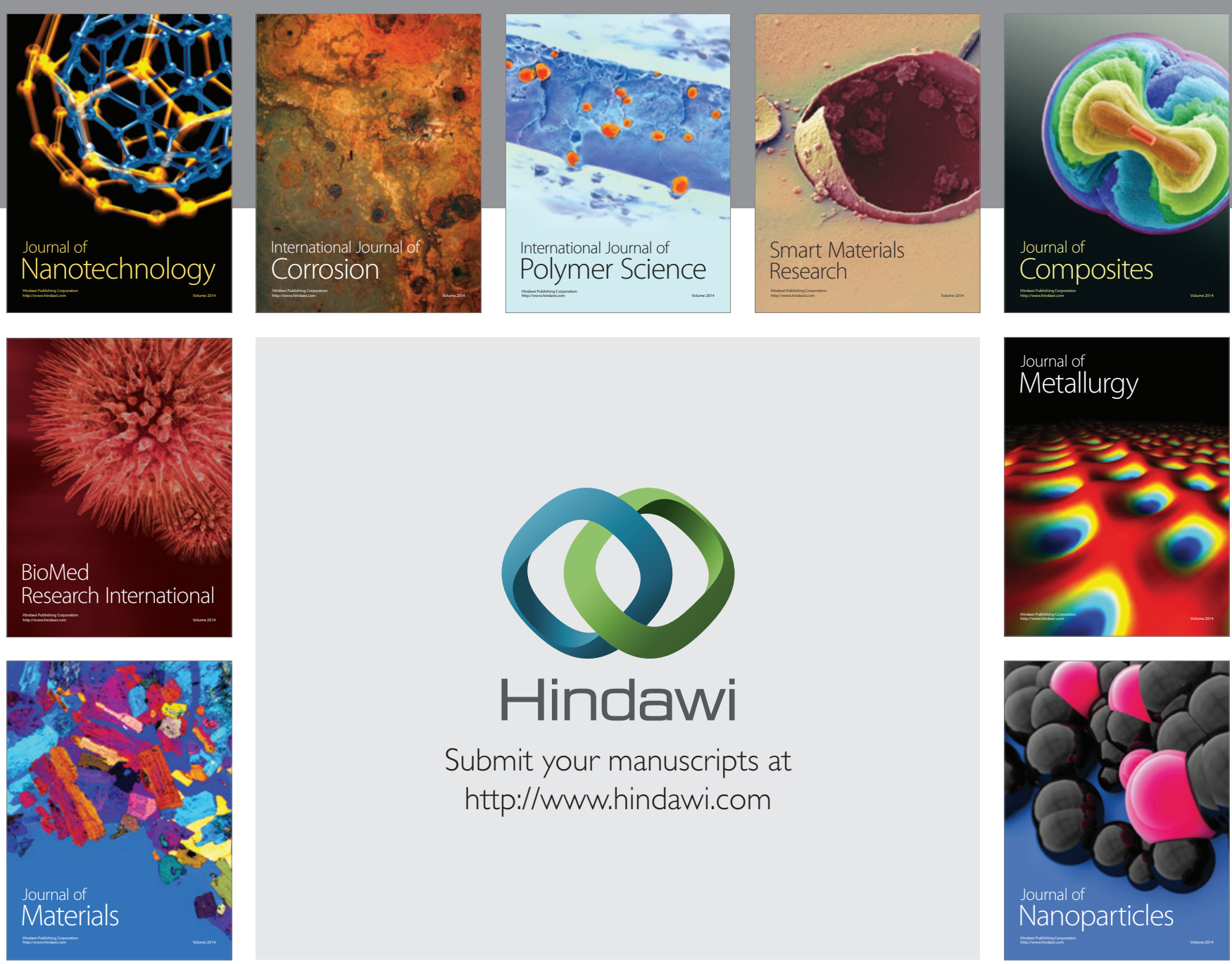

Submit your manuscripts at http://www.hindawi.com
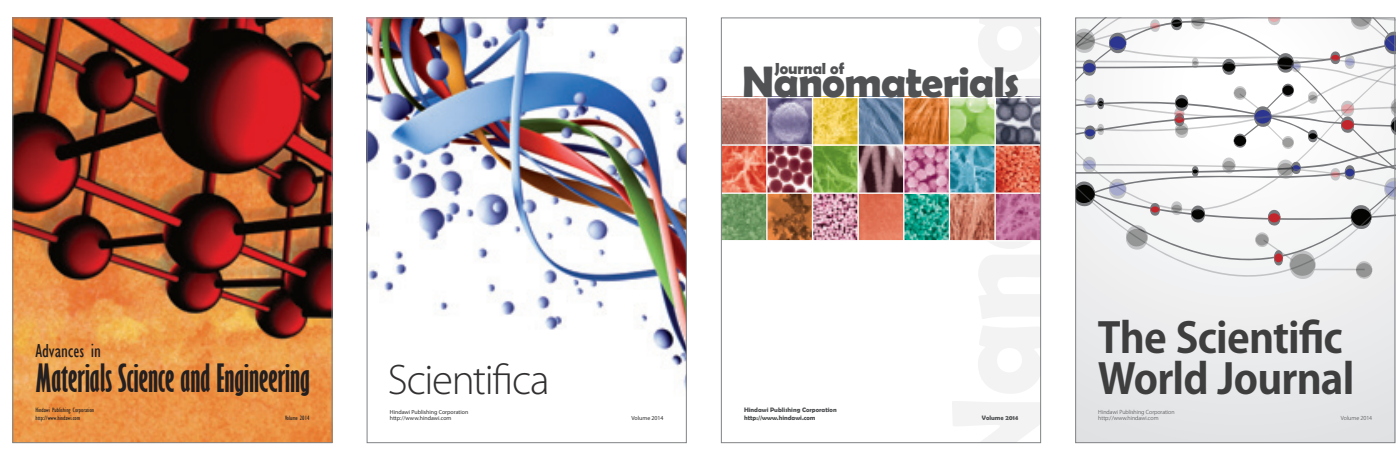

\section{The Scientific World Journal}
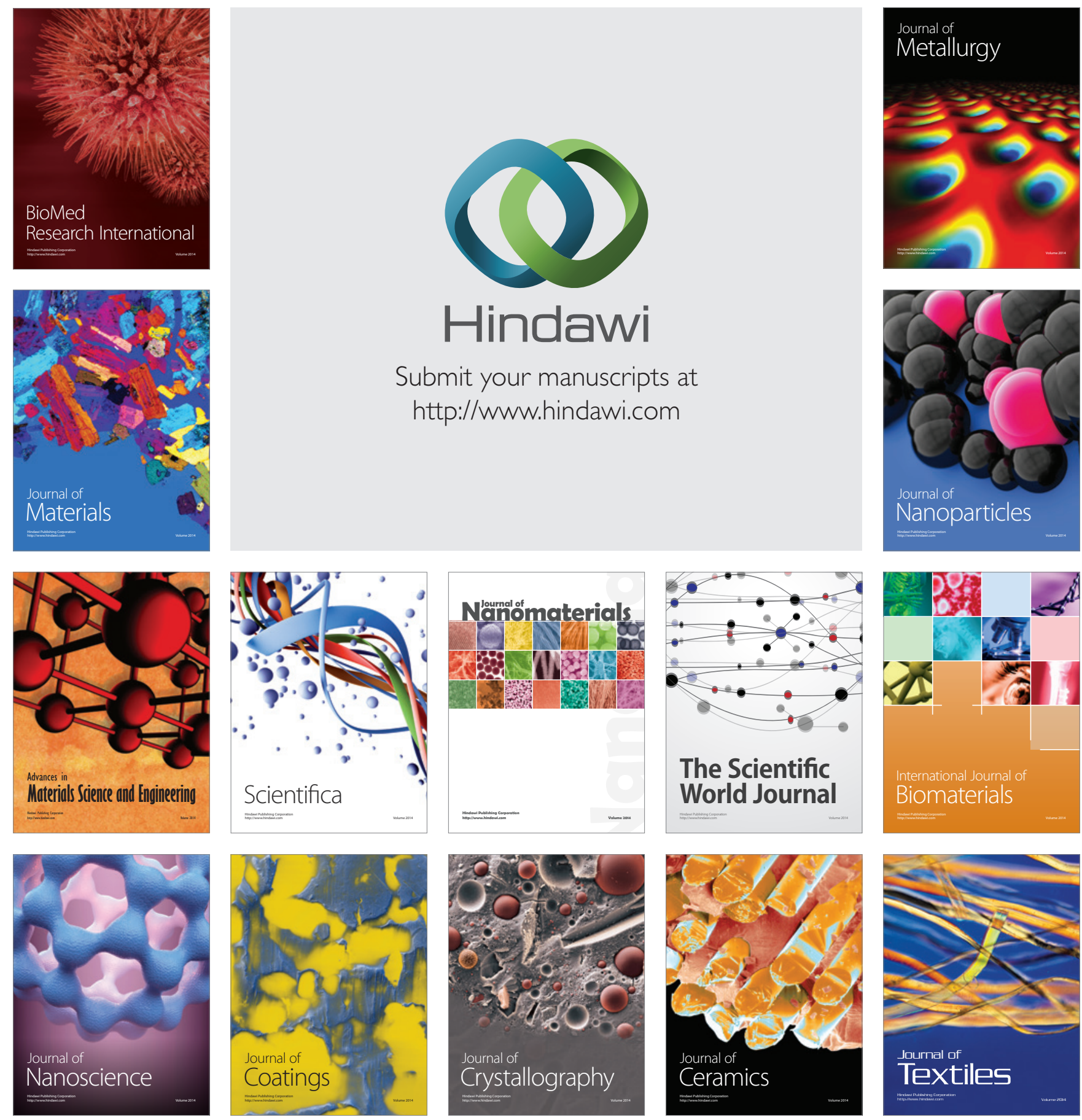